\title{
Parallel on-axis holographic phase microscopy of biological cells and unicellular microorganism dynamics
}

\author{
Natan T. Shaked, ${ }^{1, *}$ Thomas M. Newpher, ${ }^{2}$ Michael D. Ehlers, ${ }^{2,3}$ and Adam Wax ${ }^{1}$ \\ 'Department of Biomedical Engineering, Fitzpatrick Institute for Photonics, Duke University, \\ Durham, North Carolina 27708, USA \\ ${ }^{2}$ Department of Neurobiology, Duke University, Durham, North Carolina 27708, USA \\ ${ }^{3}$ Howard Hughes Medical Institute, Duke University Medical Center, \\ Durham, North Carolina 27708, USA \\ *Corresponding author: natan.shaked@duke.edu
}

Received 11 January 2010; revised 11 March 2010; accepted 19 April 2010; posted 19 April 2010 (Doc. ID 122050); published 14 May 2010

\begin{abstract}
We apply a wide-field quantitative phase microscopy technique based on parallel two-step phase-shifting on-axis interferometry to visualize live biological cells and microorganism dynamics. The parallel on-axis holographic approach is more efficient with camera spatial bandwidth consumption compared to previous off-axis approaches and thus can capture finer sample spatial details, given a limited spatial bandwidth of a specific digital camera. Additionally, due to the parallel acquisition mechanism, the approach is suitable for visualizing rapid dynamic processes, permitting an interferometric acquisition rate equal to the camera frame rate. The method is demonstrated experimentally through phase microscopy of neurons and unicellular microorganisms. () 2010 Optical Society of America

OCIS codes: $\quad 090.1995,090.2880,180.3170,170.3880$.
\end{abstract}

\section{Introduction}

Certain processes of biological cells and microorganisms, such as cell membrane fluctuations, cell swelling, movement-related phenomena, and neuronal action potentials, occur over millisecond to second time scales [1-3]. Visualizing these fast dynamic phenomena requires microscopy techniques that can achieve high data acquisition rates, while retaining spatial resolution and contrast to enable observation of fine cellular features. However, biological cells are mostly transparent, three-dimensional objects with absorbance and reflection characteristics similar to their surroundings; thus, conventional intensity-based light microscopy approaches lack the required sensitivity. As a solution, contrast agents, such as fluorescent dyes, are used. However, fluores-

0003-6935/10/152872-07\$15.00/0

(C) 2010 Optical Society of America cent contrast agents tend to photobleach, reducing the available imaging time. Other concerns include potential cytotoxicity and the possibility that the agents will influence the cellular behavior. As an alternative, phase microscopy can provide label-free information on the cellular structure and dynamics.

Traditional phase microscopy methods, such as phase contrast and differential interference contrast microscopy, are widely used today. However, these approaches also present drawbacks, including distinctive imaging artifacts. These approaches are not inherently quantitative methods and do not enable interpretation of the resulting phase images in terms of quantitative optical path delays. Wide-field digital interferometry (WFDI), on the other hand, has the potential to provide a powerful, noninvasive tool for quantitative phase measurements of biological cell dynamics [4-7]. WFDI is based on measuring the interference between a light field that has inter- 
acted with a sample and a mutually coherent reference field. With this approach, the entire sample wavefront is captured. From the recorded complex field, one can digitally reconstruct the quasi-threedimensional distribution of the sample field without the need for mechanical scanning.

Nevertheless, the interferometric signal contains unwanted dc and twin-image diffracted waves, which must be separated from the desired sample field. Off-axis interferometry [5] copes with this problem by imposing a large angle between the reference and sample beams, which creates a spatial separation between the desired and undesired waves. However, this approach does not make efficient use of the camera spatial bandwidth, which, for an image interferogram, means that high spatial frequencies in the sample field might be lost. When imaging biological dynamic processes, the need for high frame rates is frequently satisfied by using less digital camera pixels per frame, while further reducing the camera spatial bandwidth. One solution to this problem is to use cameras with higher pixel counts. However, this comes at the expense of reduced temporal resolution, limiting the acquisition rate of the dynamic process.

A different approach to removing the dc and twinimage holographic artifacts is on-axis interferometry [4]. In this approach, the angle between the sample and reference beams is set to zero, which reduces the required camera spatial bandwidth to the same as that needed for acquiring the sample intensity image alone. However, this causes the undesired diffracted waves to occlude the desired sample field. The traditional solution to this problem is to acquire three or four phase-shifted on-axis interferograms of the same sample and to separate the sample field via digital processing. This may not be practical for imaging certain dynamic processes, where the sample may change between the frame acquisitions. In addition, phase noise may increase due to system fluctuations between the frames [8]. Methods for acquiring all on-axis interferograms in a single camera exposure have been proposed (e.g., [9] and others). However, because at least three interferograms are needed, the camera spatial bandwidth [10] is still not effectively utilized. Two-step on-axis WFDI, in which two (rather than three) on-axis interferograms are required, has been suggested in [11]. However, this technique requires offline measurements of both the reference and sample fields, which is unsuitable for acquiring dynamic processes. Meng et al. [12] suggested two-step phase-shifting on-axis WFDI that requires only two interferograms and an offline reference-wave intensity measurement. Awatsuji et al. [13] demonstrated how to parallelize this technique for nonbiological, nonmicroscopic, and amplitude objects.

In this paper, we present a different parallel twostep on-axis WFDI approach, which requires only a single camera exposure for each instance of the dynamic process. Based on this approach, we employ a parallel holographic microscopy scheme to measur- ing the quantitative phase profile of cell dynamics and demonstrate, both theoretically and experimentally, that the suggested technique is more cost effective in utilizing the camera spatial bandwidth than off-axis interferometry. The new approach of parallel on-axis interferometry, coined as PONI, enables the same time resolution as off-axis interferometry, while doubling the camera spatial bandwidth consumption and maximizing the amount of spatial information that can be captured from the sample. When camera spatial bandwidth is intentionally limited to obtain high frame rates (for example, by binning or subsampling in the hardware level), camera spatial bandwidth consumption is an important factor. Thus, the parallel on-axis approach used here might be preferred to the widely used off-axis approach for visualizing highly dynamic biological processes.

\section{Methodology}

\section{A. Optical System for Obtaining Two Phase-Shifted} On-Axis Interferograms in Parallel

Figure 1 illustrates the design of the PONI quantitative phase microscope suitable for acquiring two phase-shifted on-axis interferograms in parallel. The system is composed of a modified Mach-Zehnder interferometer, followed by an image/polarization splitter. A $45^{\circ}$ linearly polarized laser light is split by beam splitter $\mathrm{BS}_{1}$ into two beams. One beam is transmitted through the biological sample and magnified by the microscope objective. The other beam serves as a reference beam and is transmitted through a quarter-wave plate, creating circular polarization, and then magnified by a similar objective lens. The beams are combined by beam splitter $\mathrm{BS}_{2}$,

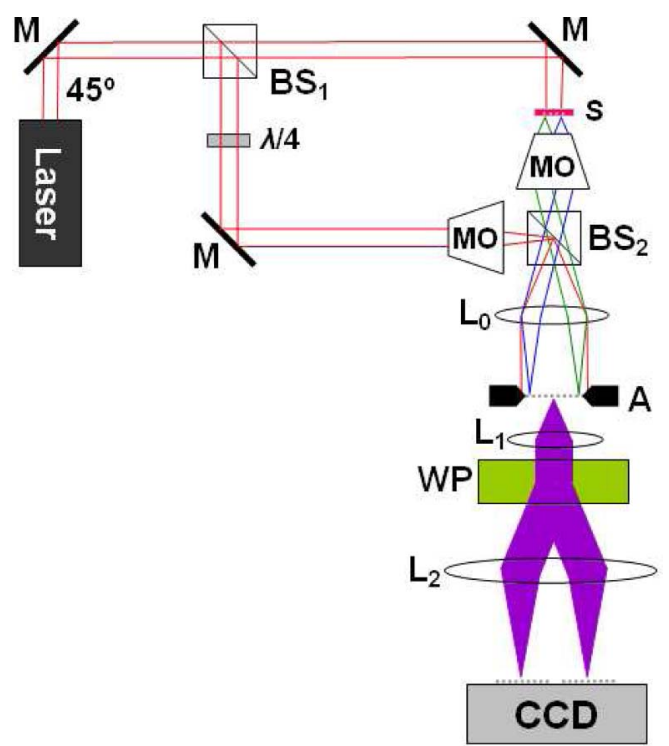

Fig. 1. (Color online) PONI microscope for quantitative phase measurements of live cell and microorganism dynamics: $\mathrm{BS}_{1}$ and $\mathrm{BS}_{2}$, beam splitters; $\mathrm{M}$, mirror; $\lambda / 4$, quarter-wave plate; $\mathrm{S}$, sample; A, rectangular aperture; $\mathrm{MO}$, microscope objective; $\mathrm{L}_{0}, \mathrm{~L}_{1}$, and $\mathrm{L}_{2}$, lenses; WP, Wollaston prism. 
where there is no angular offset between the beams. Lens $\mathrm{L}_{0}$ is in a $4 f$ configuration with both microscope objectives, imaging the phase and amplitude of the sample field in the aperture plane (A). This rectangular aperture ensures that only half of the digital camera plane is illuminated by each interferogram. The image/polarization splitter, lying beyond the aperture, includes an additional $4 f$ subsystem that images the field at the aperture plane onto the camera sensor. A Wollaston prism, positioned in the confocal plane of lenses $\mathrm{L}_{1}$ and $\mathrm{L}_{2}$, is used to split the beam into two perpendicularly polarized beams. These two beams create two on-axis interferograms at the camera, phase-shifted by $90^{\circ}$ compared to each other. This system is similar to that presented by us in [6], which used the slightly off-axis interferometric approach. In the current paper, we show that using on-axis geometry with this parallel interferometric system and a different phase retrieval algorithm provides a unique advantage by maximizing the spatial information that can be obtained per a single exposure. As noted above, this advantage is especially useful when the spatial bandwidth of the camera is intentionally limited to increase the camera frame rate.

\section{B. Sample-Field Phase Retrieval}

The two $90^{\circ}$ phase-shifted on-axis interferograms, which are acquired in a single camera exposure, can be mathematically expressed as follows:

$$
\begin{aligned}
& I_{1}=I_{R}+I_{S}+2 \sqrt{I_{R} I_{S}} \cos \left(\phi_{\mathrm{OBJ}}+\phi_{C}\right) ; \\
& I_{2}=I_{R}+I_{S}+2 \sqrt{I_{R} I_{S}} \sin \left(\phi_{\mathrm{OBJ}}+\phi_{C}\right),
\end{aligned}
$$

where $I_{R}$ and $I_{S}$ are the reference and sample intensity distributions, respectively; $\phi_{\mathrm{OBJ}}$ is the spatially varying phase of the sample; and $\phi_{C}$ is the spatially varying background phase, without the presence of the sample. Let us define $I_{0}=I_{R}+I_{S}$ and the overall phase as $\varphi=\phi_{\mathrm{OBJ}}+\phi_{C}$. It can be shown $[12,14]$ that if the reference field is strong enough compared to the sample field, $I_{0}$ can be found as one of the solutions to a quadratic equation, as follows:

$I_{0}=\frac{I_{1}+I_{2}+2 I_{R}-\sqrt{\left(I_{1}+I_{2}+2 I_{R}\right)^{2}-2\left(I_{1}^{2}+I_{2}^{2}+4 I_{R}^{2}\right)}}{2}$.

Note that in Eq. (2), $I_{0}$ can be obtained without direct knowledge of the sample intensity $I_{S}$. Thus, this procedure is valid even if $I_{S}$ changes during the observation of the dynamic process. The only a priori knowledge that is needed is an offline measurement of the reference intensity $I_{R}$, which is assumed to stay constant during the observation period. The dynamic process measurements are then performed by simultaneous recordings of two $90^{\circ}$ phase-shifted on-axis interferograms $\left(I_{1}, I_{2}\right)$, using the system illustrated in Fig. 1.
In practice, the phase retrieval procedure should include a static phase referencing step where the background phase is measured [15]. The complete phase retrieval procedure is defined as follows. In the first step, three offline measurements are taken without the presence of the sample: the sample arm intensity $I_{S}^{\prime}$; the reference arm intensity $I_{R}^{\prime}$; and two interferograms $\left(I_{1}^{\prime}, I_{2}^{\prime}\right)$. Using this data, the wrapped background phase $\tilde{\phi}_{C}$ can be calculated as follows:

$$
\begin{aligned}
& F^{\prime}=\left[\left(I_{1}^{\prime}-I_{S}^{\prime}-I_{R}^{\prime}\right)+j\left(I_{2}^{\prime}-I_{S}^{\prime}-I_{R}^{\prime}\right)\right] / \sqrt{2 I_{R}^{\prime}} ; \\
& \tilde{\phi}_{C}=\arctan \left(\operatorname{Im} F^{\prime} / \operatorname{Re} F^{\prime}\right) .
\end{aligned}
$$

Then, an unwrapping algorithm is applied to solve $2 \pi$ ambiguities in $\phi_{C}$, which yields $\phi_{C}$.

The sample is then placed in the sample arm (see Fig. 1). During the recording of the sample dynamics and for each sample observation time point, a pair of interferograms $\left(I_{1}, I_{2}\right)$ is continuously recorded, where each pair is recorded in a single camera exposure. Next, the overall wrapped phase is calculated as follows:

$$
\begin{aligned}
F & =\left[\left(I_{1}-I_{0}\right)+j\left(I_{2}-I_{0}\right)\right] / \sqrt{2 I_{R}} \\
\tilde{\varphi} & =\arctan (\operatorname{Im} F / \operatorname{Re} F),
\end{aligned}
$$

where $I_{R}=I_{R}^{\prime}$ and, thus, is already known. An unwrapping algorithm is applied to solve $2 \pi$ ambiguities in $\tilde{\varphi}$, which yields $\varphi$. Finally, the sample phase is calculated as follows:

$$
\phi_{\mathrm{OBJ}}=\varphi-\phi_{C} .
$$

Because the information for each observation of the sample is acquired in a single camera exposure, the method itself is not limited to the rate in which the dynamic process changes, and the only limiting factor here is the true frame rate of the digital camera used.

\section{Theoretical and Experimental Comparison to the Traditional Off-Axis Approach}

The spatial-frequency representation of a typical interferogram [for example, each one of the interferograms mathematically defined in Eq. (1)] contains four terms: two dc terms that arise from $I_{R}$ and $I_{S}$, and two high-order (image and twin-image) terms that arise from the modulation element [either from the cosine or the sine in Eq. (1)] [16]. To reconstruct the sample wavefront, only one of the high-order terms is needed. Thus, interferometric methods seek to separate the desired term from the other three undesired terms. Off-axis interferometry solves this problem by imposing a large angle between the reference and sample arms, creating a spatial-frequency separation between the wanted and unwanted terms. However, this comes at the expense of losing spatial bandwidth on the camera, basically because many camera pixels are needed to acquire the 
high-frequency fringes that create the spatialfrequency separation. As illustrated in Fig. 2(a), the requirement for the spatial-frequency separation for an off-axis image interferogram causes the highest spatial frequency required on the camera to be four times the highest frequency of the sample $\omega_{0}$ (along the axis of the off-axis angle). Despite this disadvantage, off-axis interferometry is considered the leading technique for interferometric recording of dynamic processes because the required information can be acquired in a single camera exposure.

In the on-axis geometry, on the other hand, there is no angle between the reference and sample beams. Thus, the camera spatial bandwidth consumption is more effective because now all four terms are centered at the origin of the spatial-frequency domain [Fig. 2(b)]. However, the problem of separating the desired terms from the unwanted terms is solved by acquiring multiple phase-shifted interferograms of the same sample. If these interferograms are acquired in sequence, the sample itself or even the system noise might change during the acquisition time points.

Projecting several phase-shifted on-axis interferograms on the same camera can be used as a means to overcome this drawback. However, because three or four phase-shifted interferograms are needed in traditional on-axis approaches, the highest spatial frequency (and the spatial bandwidth) required from the camera is equivalent to at least $3 \omega_{0}$ or $4 \omega_{0}$, respectively. Because the method proposed in this paper requires only two phase-shifted interferograms, the highest spatial frequency on camera is equivalent to $2 \omega_{0}$ [Fig. 2(b)], half of that required for the off-axis case [Fig. 2(a)].

Before presenting the biological-sample experimental results in Section $\underline{4}$, we first provide two simple experimental demonstrations using a static USAF test target. In the first experiment, we have imaged groups 4-7 of the test target in low magnification. We have used a $17 \mathrm{~mW} \mathrm{He}-\mathrm{Ne}$ laser and CCD camera (Pike F-032B, Allied Vision Technologies, Germany) that has a pixel size of $7.4 \mu \mathrm{m} \times 7.4 \mu \mathrm{m}$ and allows several binning and subsampling options at the hardware level. Binning and subsampling of the camera pixels is frequently performed to increase the temporal frame rate of the digital camera and thus to enable the acquisition of faster dynamic processes, as demonstrated in Section $\underline{4}$.

For the on-axis phase-shifting method, we have used $0.54 \times$ magnification, which means that the smal-
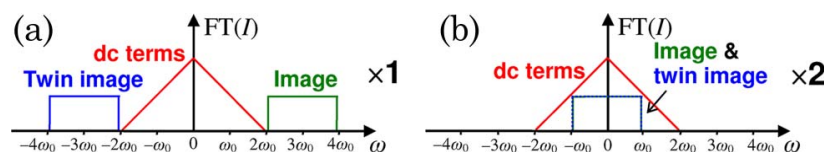

Fig. 2. (Color online) Schematic representation of the spatialfrequency domain of (a) the off-axis method (acquisition of a single interferogram) along the axis of the off-axis angle, and (b) the proposed PONI method (simultaneous acquisition of two one-axis interferograms). FT: spatial Fourier transform. lest resolvable detail of the test target due to the camera pixel size is group 5 element $2(36 \mathrm{lp} / \mathrm{mm})$. Two phase-shifted on-axis interferograms of the test target were acquired and processed according to the algorithm described in Section 2, with the resulting reconstructed amplitude distribution shown in Fig. 3(a). A single off-axis interferogram of the same groups of the test target was acquired with $1.08 \times$ magnification and $2 \times$ horizontal binning, while inducing the maximal off-axis angle possible in the vertical dimension, with the resulting reconstructed amplitude distribution shown in Fig. 3(b). Note that in the offaxis case a single interferogram (rather than two) is required; therefore, the entire camera sensor size can be used per interferogram (and not half of the sensor size as in the parallel on-axis case). Thus, to implement an equal comparison to the singleinterferogram off-axis case and to keep the camera spatial bandwidth consumption constant, in the offaxis case we have used twice the magnification used in the on-axis case. Then, to compensate for the fact that the magnification operates in both dimensions, one-dimensional binning is performed in the off-axis case. In spite of the fact that both methods consume the same effective camera spatial bandwidth, it is obvious from Fig. 3 that the on-axis geometry can resolve finer details in the vertical dimension compared to the traditional off-axis case due to the more effective use of the camera spatial bandwidth in the vertical dimension.

Note that for this demonstration and for all other demonstrations in the paper, even though we do not present the entire field of view recorded on the camera, the same part of the recorded field of view is presented for the on-axis and off-axis cases, so that the same effective spatial bandwidth of the camera is still used in both cases.
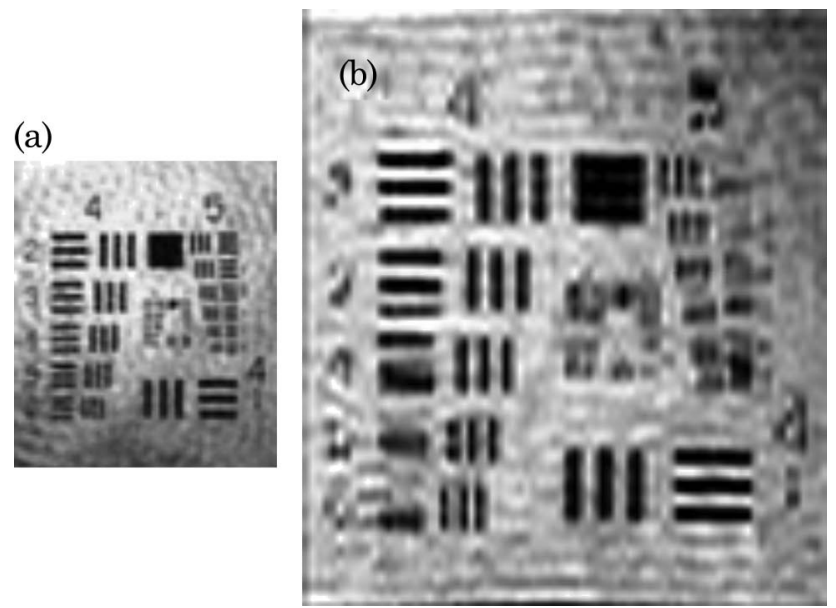

Fig. 3. Reconstructed amplitude images of groups 4-7 of USAF resolution target obtained by (a) the suggested on-axis method, $0.54 \times$ magnification, no binning, and (b) the traditional off-axis method, $1.08 \times$ magnification, $2 \times$ horizontal binning. The off-axis angle is along the vertical dimension [where resolution loss is seen compared to (a)]. 
The second demonstration includes imaging of group 7, elements 2-6 of the USAF test target under high magnification. We have used a 0.66 numerical-aperture microscope objective, permitting a diffraction-limited spot of $0.48 \mu \mathrm{m}$. For the on-axis experiment, we have formed a $33 \times$ total magnification by coupling the microscope objective with a $15 \mathrm{~cm}$ lens in $4 f$ configuration. Under this magnification, the diffraction-limited spot of the optics projected onto the camera is $15.8 \mu \mathrm{m}$ in size, occupying approximately 2 camera pixels. However, the smallest line pair in the sample (group 7, element 6 in the test target, $228 \mathrm{lp} / \mathrm{mm}$ ) projected onto the camera occupies about 20 camera pixels, whereas only 2 pixels are truly needed to acquire this line pair. Thus, for this sample and under this magnification, there in no need to use all camera pixels, and pixel binning can be performed to consume the camera spatial bandwidth more efficiently. We have performed $8 \times 8$ binning, which effectively creates a camera pixel size of $59 \mu \mathrm{m} \times 59 \mu \mathrm{m}$, without loss of the smallest detail (thinnest line) of the test target. We have first acquired two phaseshifted on-axis interferograms of the test target and digitally process them as explained in Section 2 , with the reconstructed amplitude distribution presented in Fig. 4(a). Then, the same group and elements of the test target were imaged using traditional off-axis geometry, inducing the largest off-axis angle possible in the vertical dimension under $8 \times 8$ camera pixel binning. Because the off-axis geometry requires only one interferogram, we have created a total optical magnification of $66 \times$ onto the camera. The reconstructed amplitude distribution obtained from this off-axis interferogram is shown in Fig. 4(b), demonstrating again the improvement of resolution in the vertical dimension that can be gained, with the same limited camera spatial bandwidth consumption in the vertical dimension, by using two on-axis interferograms rather than one off-axis interferogram.

\section{Experimental Results of Biological Samples}

For constructing the optical system shown in Fig. 1, we have used again the 0.66 numerical-aperture microscope objectives. The focal length of $\mathrm{L}_{0}$ was $15 \mathrm{~cm}$, and the focal lengths of lenses $\mathrm{L}_{1}$ and $\mathrm{L}_{2}$ were $7.5 \mathrm{~cm}$ each, where the total magnification of the system was $33 \times$. Again, the diffraction-limited spot was (a)

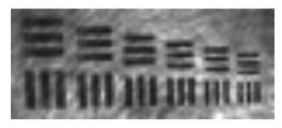

(b)

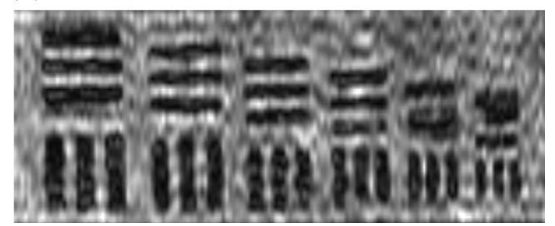

Fig. 4. Reconstructed amplitude images of groups 7, elements 26 of USAF resolution target obtained by (a) the suggested on-axis method, 33× magnification, $8 \times 8$ binning, and (b) the traditional off-axis method, $66 \times$ magnification, $8 \times 8$ binning. The off-axis angle is along the vertical dimension [where resolution loss is seen compared to (a)].
$0.48 \mu \mathrm{m}$ in size $(15.9 \mu \mathrm{m}$ when projected onto the digital camera). Note that each diffraction-limited spot must be captured by at least 2 camera pixels to avoid loss of resolution of the optics. For comparison purposes, we have also implemented a typical off-axis setup. This was accomplished by removing the waveplate and the Wollaston prism, inducing a large angle between the reference and sample beams, changing lens $\mathrm{L}_{2}$ to another lens with a focal length of $15 \mathrm{~cm}$ (instead of $7.5 \mathrm{~cm}$ ), and shifting the camera accordingly to retain the $4 f$ configuration of lenses $\mathrm{L}_{1}$ and $\mathrm{L}_{2}$. The replacement of lens $\mathrm{L}_{2}$, which results in a doubling of the total magnification of the optical system, allows full utilization of the digital camera imaging area because, for the off-axis case, only one interferogram is needed. To make this comparison valid, the same sample focus conditions were kept and, for the off-axis case, the angle between the reference and sample beams was made large enough in the horizontal dimension to take advantage of the full available camera spatial bandwidth in this dimension. In addition, in the off-axis case, $2 \times$ camera pixel binning was performed in the vertical dimension to keep the camera spatial bandwidth consumption the same as that of the parallel-on-axis case, in spite of the symmetric magnification used.

We have first experimentally demonstrated the proposed method by imaging a fixed rat hippocampal neuron in phosphate buffered saline (PBS). This sample was chosen because it contains small spatial details outside of the cell body, the neuronal axons and the dendrites. Prior to the experiment, hippocampal neuron cultures were prepared from E18 rat embryos and maintained for 17 days in vitro as described in [17]. For fixation, hippocampal neurons were placed in a solution containing $4 \%$ paraformaldehyde/4\% sucrose in PBS for 15 min.

Figure 5(a) shows two $90^{\circ}$ phase-shifted, on-axis interferograms of the neuron acquired in a single camera exposure. Figure 5(b) shows the phase profile of this neuron, obtained by processing the two interferograms shown in Fig. 5(a) according to the digital process explained in Subsection 2.B. Figure 5(c) shows the phase profile obtained by the off-axis technique. Despite the fact that this phase image is double in size across the horizontal dimension (because in the off-axis case the entire sensor area is used for each interferogram), there is a loss of spatial resolution compared to the proposed method [Fig. 5(b)] for the reasons explained in Section 3.

For the second experimental demonstration of phase microscopy of biological samples, we have chosen a dynamic unicellular protist called Euglena gracilis in water as the sample. This sample was chosen due to the Euglena's flagellum, the thin tail used for propulsion, which is located outside the Euglena's body. The thin flagellum of the Euglena moves at the millisecond time scale, and thus should be observed with a WFDI technique that loses neither spatial nor time resolution. To be able to demonstrate a loss of resolution when imaging the Euglena's thin 

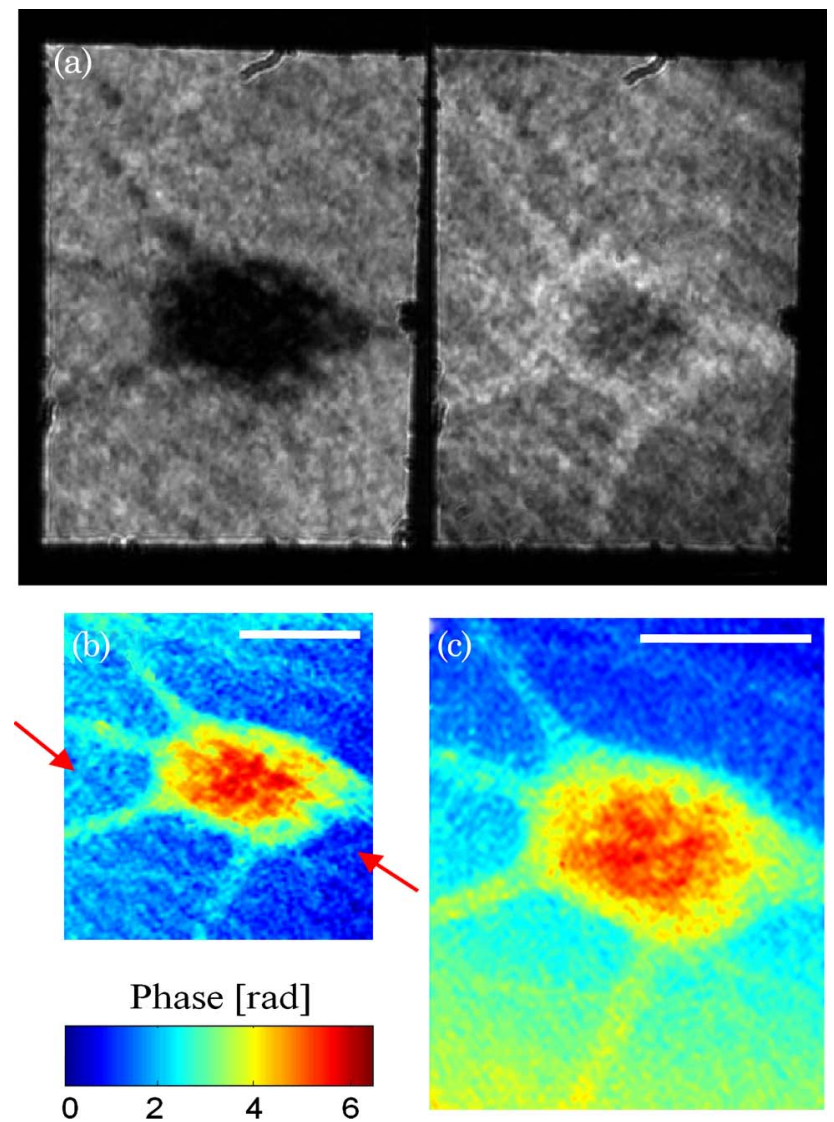

Fig. 5. (Color online) Rat hippocampal neuron: (a) two phaseshifted on-axis interferograms acquired in a single camera exposure; (b) unwrapped phase profile obtained by the suggested PONI method, 33× magnification, no binning; (c) unwrapped phase profile obtained by the traditional off-axis method, $66 \times$ magnification, $2 \times$ vertical binning. The white scale bars indicate $5 \mu \mathrm{m}$. Phase colorbar is valid for both (b) and (c). In spite of the fact that (b) is half in size compared to (c), finer details can be detected in (b). Red arrows in (b) indicate two examples for that.

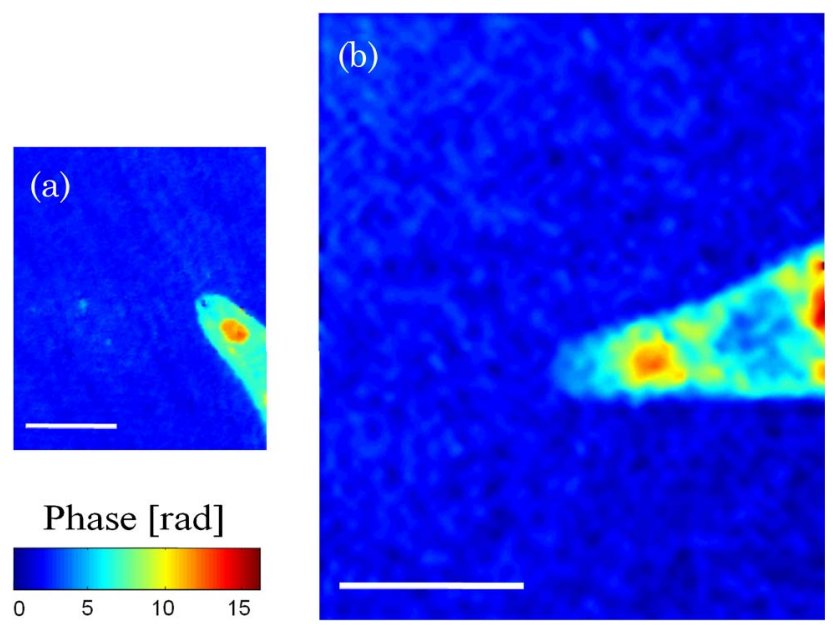

Fig. 6. (Color online) Upper part of Euglena gracilis (a unicellular protist): (a) unwrapped phase profile obtained by the suggested PONI method, 33× magnification, $2 \times 2$ binning (dynamic behavior: Media 1); (b) unwrapped phase profile obtained by the traditional off-axis method, $66 \times$ magnification, $4 \times 2$ binning (dynamic behavior: Media 2). The white scale bars indicate $10 \mu \mathrm{m}$. Phase colorbar is valid for both (a) and (b). flagellum by the traditional off-axis approach, as compared to the proposed parallel on-axis case, the phase profile of the sample has been acquired by the proposed (2-interferogram) on-axis technique with $2 \times 2$ camera pixel binning and $33 \times$ magnification, and by the traditional (1-interferogram) off-axis technique with $4 \times 2$ camera pixel binning and $66 \times$ magnification. As in the previous demonstrations, the same camera spatial bandwidth has been used in both cases. The final phase images are shown in Figs. 6(a) and 6(b) for the proposed PONI and for the traditional off-axis methods, respectively. The flagellum dynamics are presented in Media 1 and Media 2, demonstrating the high temporal acquisition rate, as well as the spatial-resolution improvement obtained by the proposed method compared to the traditional off-axis case.

\section{Conclusion}

We have presented the PONI, a modification of our parallel WFDI method that is useful for capturing the quantitative phase profiles of the dynamics of optically transparent biological cells and unicellular organisms containing microscopic spatial details. Using this method, we show that the spatial bandwidth of the camera is utilized more optimally, compared to the traditional off-axis interferometry, while still maximizing the temporal resolution by acquiring the required interferometric information in a single exposure. The method has been experimentally demonstrated by phase imaging of the fine features of dynamic Euglena gracilis, a unicellular microorganism, and a static hippocampal neuron. WFDI phase microscopy at fast frame rates and high spatial resolution might permit recording the changes in the index of refraction occurring during neuronal action potential propagation, as will be explored in our future work.

This work was supported by National Science Foundation (NSF) grants CBET-0651622 and BES 03-48204. N. Shaked gratefully acknowledges the support of the Bikura Postdoctoral Fellowship from Israel.

\section{References}

1. L. H. Deng, X. Trepat, J. P. Butler, E. Millet, K. G. Morgan, D. A. Weitz, and J. J. Fredberg, "Fast and slow dynamics of the cytoskeleton," Nat. Mater. 5, 636-640 (2006).

2. M. W. Barnett and P. M. Larkman, "The action potential," Pract. Neurol. 7, 192-197 (2007).

3. D. Bray, Cell Movements (Garland, 1992).

4. G. Popescu, L. P. Deflores, J. C. Vaughan, K. Badizadegan, H. Iwai, R. R. Dasari, and M. S. Feld, "Fourier phase microscopy for investigation of biological structures and dynamics," Opt. Lett. 29, 2503-2505 (2004).

5. P. Marquet, B. Rappaz, P. J. Magistretti, E. Cuche, Y. Emery, T. Colomb, and C. Depeursinge, "Digital holographic microscopy: a noninvasive contrast imaging technique allowing quantitative visualization of living cells with subwavelength axial accuracy," Opt. Lett. 30, 468-470 (2005).

6. N. T. Shaked, M. T. Rinehart, and A. Wax, "Dual-interferencechannel quantitative-phase microscopy of live cell dynamics," Opt. Lett. 34, 767-769 (2009). 
7. N. T. Shaked, J. D. Finan, F. Guilak, and A. Wax, "Quantitative phase microscopy of articular chondrocyte dynamics by widefield digital interferometry," J. Biomed. Opt. 15, 010505 (2010).

8. C. P. Brophy, "Effect of intensity error correlation on the computed phase of phase-shifting interferometry," J. Opt. Soc. Am. A 7, 537-541 (1990).

9. Y. Awatsuji, A. Fujii, T. Kubota, and O. Matoba, "Parallel three-step phase-shifting digital holography," Appl. Opt. 45, 2995-3002 (2006).

10. A. Stern and B. Javidi, "Space-bandwidth conditions for efficient phase-shifting digital holographic microscopy," J. Opt. Soc. Am. A 25, 736-741 (2008).

11. P. Guo and A. J. Devaney, "Digital microscopy using phaseshifting digital holography with two reference waves," Opt. Lett. 29, 857-859 (2004).

12. X. F. Meng, L. Z. Cai, X. F. Xu, X. L. Yang, X. X. Shen, G. Y. Dong, and Y. R. Wang, "Two-step phase-shifting interferometry and its application in image encryption," Opt. Lett. 31, 1414-1416 (2006).
13. Y. Awatsuji, T. Tahara, A. Kaneko, T. Koyama, K. Nishio, S. Ura, T. Kubota, and O. Matoba, "Parallel two-step phaseshifting digital holography," Appl. Opt. 47, D183-D189 (2008).

14. J.-P. Liu and T.-C. Poon, "Two-step-only quadrature phaseshifting digital holography," Opt. Lett. 34, 250-252 (2009).

15. P. Ferraro, S. De Nicola, A. Finizio, G. Coppola, S. Grilli, C. Magro, and G. Pierattini, "Compensation of the inherent wave front curvature in digital holographic coherent microscopy for quantitative phase-contrast imaging," Appl. Opt. 42, 19381946 (2003).

16. N. T. Shaked, Y. Zhu, M. T. Rinehart, and A. Wax, "Two-steponly phase-shifting interferometry with optimized camera bandwidth for microscopy of live cells," Opt. Express 17, 15585-15591 (2009).

17. M. D. Ehlers, "Reinsertion or degradation of AMPA receptors determined by activity-dependent endocytic sorting," Neuron 28, 511-525 (2000). 